\title{
Effect of different toxic compounds on ATP content and acid phosphatase activity in axenic cultures of Tetrahymena pyriformis
}

\author{
Ana Nicolau, Manuel Mota, and Nelson Lima* \\ Centro de Engenharia Biológica, Universidade do Minho, Campus de Gualtar, Braga 4710-057, Portugal
}

Received 6 June 2002; received in revised form 16 September 2003; accepted 8 October 2003

\begin{abstract}
The sensitivity of protozoa, and particularly ciliated protozoa, to environmental changes suggested a study on the physiological responses arising from exposure to toxic compounds. Tetrahymena pyriformis was used as a test organism in a set of miniaturized assays. The physiological response of this ciliate was assessed in terms of adenosine-5'-triphosphate content and acid phosphatase activity after exposure of the cultures of $T$. pyriformis to four toxicants: copper, zinc, Triton X-100, and cycloheximide. In the range of concentrations used, stimulation and inhibition of these two parameters were observed. The correlation between the two parameters is analyzed.
\end{abstract}

(C) 2003 Elsevier Inc. All rights reserved.

Keywords: Protozoa; Tetrahymena pyriformis; In vitro toxicity; ATP content; ACP activity

\section{Introduction}

The increasing environmental pollution and the continuous development of new drugs have led to an ever growing concern about the potential effects of these compounds on human health, directly or indirectly. Over recent years much research has been done on the toxicity of various relevant toxic compounds in a series of biotests using several test organisms. The appeal of these tests lies in their simplicity and high degree of reproducibility. On the other hand, test organisms for assessing environmental risk and impact must possess a lot of desirable features: they must be eukaryotic, their biology and general responses must be well known, the laboratory handling must be relatively easy, and a short generation time is desirable whenever studies of longterm effects are necessary (Nilsson, 1989). Ciliated protozoa fulfil all these requirements. Furthermore, as they have a wide distribution and ecological significance, performing key functions in energy flow and elementary cycling in aquatic food webs, they can be ideal early warning indicators of aquatic ecosystem deterioration (Twagilimana et al., 1998).

${ }^{*}$ Corresponding author. Fax: +351-253678986.

E-mail address: nelson@iec.uminho.pt (N. Lima).
The ciliated Tetrahymena pyriformis has been, for more than 4 decades, the organism of choice in analyses, evaluation of protein quality, and determination of effects of several toxic substances (Nicolau et al., 1999, 2001; Dias et al., 1998, 1999, 2003; Dias and Lima, 2002; Piccinni et al., 1987; Meyer et al., 1971; Nilsson, 1981; Yamaguchi et al., 1973; Yoshioka et al., 1985; Noever et al., 1994; Sauvant et al., 1994; Ward and Codd, 1999; Akers et al., 1999). Moreover, it was the first protozoon to be cultivated axenically, i.e., in a standard medium, free from bacteria or other organisms, making it a suitable model cell system since the addition of a compound can be, in principle, the only change in culture conditions.

Adenosine- $5^{\prime}$-triphosphate (ATP) is a molecule present in all viable cells, functioning as a carrier of chemical energy between catabolic reactions and all the cellular processes which require an energy input. Assays of ATP content have been widely used to characterize biomass viability (Gikas and Livingston, 1993) and to detect potential spoilage microorganisms in the beer and food industry (Gamborg and Hansen, 1994; Dowhanick and Sobczak, 1994), assuming that it can be possible to use the concentration of ATP as a measure of viable cells of a certain species. In the present work, ATP content was determined to give information about the 
general energetic state of the culture, when submitted to the toxicants.

Previous works have been devoted to the study of acid phosphatase (ACP) activity and of other hydrolases to detect digestive activity in protozoa (Rothstein and Blum, 1974a, b; Ricketts and Rappit, 1975; Williams and Juo, 1976), since there is an intimate relationship between lysosomal function and intracellular digestion in Tetrahymena and other ciliates but only a few authors suggested the assessment of hydrolase activity to evaluate toxicity (Martin and Clydes, 1991). In the present work, ACP activity was used as an indicator of the metabolic state of the cultures, namely of the intracellular digestive function.

In order to see if there are any interactions between these parameters, $T$. pyriformis was exposed to different concentrations of copper, zinc, cycloheximide (an antibiotic), or Triton X-100 (a neutral surfactant) in a set of miniaturized assays in which ATP content and ACP activity of the cultures were evaluated simultaneously. The novelty of the study lies precisely in the fact that ATP content and ACP activity are studied simultaneously, under the same experimental conditions, making possible the search for interactions between the two parameters.

\section{Materials and methods}

\subsection{Organisms and media}

In all toxicological assays, axenic $18-24 \mathrm{~h}$ cultures of T. pyriformis GL were used. The culture medium used was PPY (proteose peptone yeast extract medium; Catalogue of Strains of Culture Collection of Algae and Protozoa, UK). It contains $20.0 \mathrm{~g} \mathrm{~L}^{-1}$ of proteose peptone and $2.5 \mathrm{~g} \mathrm{~L}^{-1}$ of yeast extract and its $\mathrm{pH}$ value lies between 6.6 and 6.8. All tests were performed at $20 \pm 1^{\circ} \mathrm{C}$. Initial densities of cultures reached about 5000 individuals $\mathrm{mL}^{-1}$.

\subsection{Toxicants and concentrations}

Six nominal concentrations of copper were used: 50 , 100, 200, 300, 400, and $500 \mathrm{mg} \mathrm{L}^{-1}$. Four nominal concentrations of zinc were used: 50, 100, 200, and $300 \mathrm{mg} \mathrm{L}^{-1}$. $\mathrm{CuCl}_{2} \cdot 2 \mathrm{H}_{2} \mathrm{O}$ and $\mathrm{ZnCl}_{2}$ were used to prepare the toxicant solutions. In both cases, $\mathrm{pH}$ was adjusted to values between 6.6 and 6.8 by the addition of $0.1 \mathrm{~N} \mathrm{NaOH}$. Distilled tap water, with a $\mathrm{pH}$ around 5.5, was used to prepare both medium and toxicant solutions.

Soluble concentrations of heavy metals were determined considering that only the amount of soluble metal is available, and so, only this amount is toxic to the cells. Determinations of soluble copper and zinc were obtained after dialysis of the PPY medium through a cellulose membrane of $3500 \mathrm{Da}$ (molecular weight cutoff). For each concentration, two replicates of $10 \mathrm{~mL}$ were subjected to dialysis for $24 \mathrm{~h}$ in $5 \mathrm{~L}$ of deionized tap water with $0.02 \mathrm{~g} \mathrm{~L}^{-1}$ sodium azide, at $20 \pm 1^{\circ} \mathrm{C}$. This water was renewed three times, after which both copper and zinc remaining in the medium were determined by photometric Merk Spectroquant Methods, after acid digestion with nitric acid (65\%). This was considered to be the amount of metal bound to the dissolved organic matter, allowing determination of the effectively bioavailable fraction of each metal. Less than $1 \%$ of total protein was lost during dialysis. Protein was determined by the Sigma Micro Protein Determination Method, based on the biuret and Lowry procedures.

Prior to the toxicity assays, a control assay was performed to investigate the influence of the chloride ion present in copper and zinc assays. Sodium chloride was used in this control assay. No effect was detected in any of the chloride concentrations used in the metal assays.

Triton X-100, a neutral surfactant that disrupts the cell membrane, was used at five concentrations: 12.5, 25.0, 37.5, 50.0, and $75.0 \mathrm{mg} \mathrm{L}^{-1}$. Cycloheximide, an antibiotic that inhibits protein synthesis in eukaryotic cells, was used in five concentrations: 0.0002, 0.001, $0.01,0.1$, and $1.0 \mathrm{mg} \mathrm{L}^{-1}$. The $\mathrm{pH}$ values of the culture medium did not significantly change from the addition of either of the last two toxicants.

\subsection{Toxicity assays}

Cultures of $T$. pyriformis were exposed to the toxicants for $48 \mathrm{~h}$ and assays were performed at 1, 24, and $48 \mathrm{~h}$, evaluating ATP content and ACP activity. For each toxicant, two independent assays were carried out. For each concentration tested, three replicates were made. A supplementary set of three replicates, without toxicant, was set up for each assay as a control. The incubation with the toxicants was done in 24-well tissue culture dishes. Total volume per well was $1.5 \mathrm{~mL}: 1.0 \mathrm{~mL}$ of PPY with the toxicant at the required concentration and $0.5 \mathrm{~mL}$ of inoculum of $T$. pyriformis.

Several authors have pointed out the importance of linearity between density and the parameter that is being tested to assess viability or survival of a species; this was verified for both ATP content and ACP activity of the cultures, under control conditions, before the assays with the toxicants.

ATP content was evaluated by the bioluminescence method using a Lumac Biocounter Model M2500, after exposure of the organisms to a luciferin/luciferase complex. The method is based on the liberation of light during the oxidation of luciferin by oxygen in the presence of ATP, magnesium ions, and luciferase. ATP is converted to AMP with concurrent generation of pyrophosphate and light, which is emitted near $560 \mathrm{~nm}$. 
It is assumed that the emitted light is directly proportional to the quantity of ATP available. The ATP kit used for these analyses was the Lumac PM kit, containing NBR, a nucleotide-releasing reagent, and Lumit PM, the purified freeze-dried luciferin and luciferase preparation, which is rehydrated in a diluent containing $\quad 0.025 \mathrm{M} \mathrm{N}$-2-hydroxyethylpiperazine- $N$-2ethanesulfonic acid buffer, $\mathrm{MgSO}_{4}$, EDTA, sodium azide, and a kinetic enhancer. The Lumac Biocounter M2500 was programmed to proceed as follows: $100 \mu \mathrm{L}$ of NBR was added to the cuvette in which was previously poured $100 \mu \mathrm{L}$ of sample; after 30 s to allow for the extraction of ATP, $100 \mu \mathrm{L}$ of Lumit QM was added and the mixture was gently swirled for $10 \mathrm{~s}$ after which the RLU (relative light units) were recorded. A previous assay was made to obtain a calibration curve to correlate RLU and ATP content in $\mathrm{mg} \mathrm{L}^{-1}$. The equation is

$\mathrm{RLU}=884.25+3,102,801 \times \mathrm{ATP}$

with a correlation coefficient $R=0.9988$ between 890 and 160,000 RLU. Dilutions were made to keep the RLU values within this range. Results are presented as ATP content in $\mathrm{mg} \mathrm{L}^{-1}$.

ACP activity was determined using the Sigma Kit 435-A, based on the hydrolysis of $\alpha$-naphthyl phosphate to $\alpha$-naphthol by ACP and subsequent production of the Fast Red chromophore, which can be quantified at $405 \mathrm{~nm}$. Color intensity is directly proportional to ACP activity. Colorimetric quantification was made directly in microplates, using a spectrophotometric microtiter plate reader (ELISA) - SLT Spectra 1 and following step-by-step the manufacturers instructions, adjusting only the volumes of reagents ( $\alpha$-naphthyl phosphate and Fast Red colorant) and samples to a total volume of $0.3 \mathrm{~mL}$ per well. Results are presented in terms of units of enzyme activity per liter $\left(\mathrm{U} \mathrm{L}^{-1}\right)$. One unit of activity is defined as the quantity of enzyme needed to hydrolyze one molecule of $\alpha$-naphthyl phosphate per minute under the assay conditions.

Experimental results were then analyzed by means of a correlation coefficient. The purpose of correlation analysis is to measure the intensity of association between any pair of variables and to test whether it is greater than can be explained by chance alone. When data, like the present, are known not to follow the bivariate normal distribution, a coefficient of rank correlation can be used. In the present work, Kendall's coefficient of rank correlation was used.

\section{Results}

Table 1 shows the nominal metal concentrations used and the corresponding concentrations of soluble metal. All toxicological data refer to soluble metal concentrations.
Table 1

Nominal and soluble concentrations of the heavy metal toxicants used in the present study

\begin{tabular}{lcc}
\hline $\begin{array}{l}\text { Metal nominal } \\
\text { concentration }\left(\mathrm{mg} \mathrm{L}^{-1}\right)\end{array}$ & \multicolumn{2}{c}{ Soluble metal concentration $\left(\mathrm{mg} \mathrm{L}^{-1}\right)$} \\
\cline { 2 - 3 } & Copper & Zinc \\
\hline 50 & 30 & 15 \\
100 & 65 & 45 \\
200 & 145 & 120 \\
300 & 200 & 200 \\
400 & 275 & - \\
500 & 350 & - \\
\hline
\end{tabular}

The results of the assays on ATP content and ACP activity of the cultures are shown in Fig. 1. In most cases, increasing concentrations of toxicants caused increasing inhibition of the physiological responses of $T$. pyriformis, but in some cases toxicants caused stimulation of the two parameters. Initial values of the parameters were low due to low initial densities of ciliates in the cultures, but were always over zero; in the graphs, due to the scale-down need, these initial values seem to be zero, but they are not.

Description of results emphasizes those obtained after $48 \mathrm{~h}$ of incubation, because after $24 \mathrm{~h}$, differences were less significant and they did not contradict the differences observed later. After $1 \mathrm{~h}$ incubation, almost all cultures were in the same state, though some inhibition was yet observed for the highest concentrations of toxicants.

The lowest copper concentration assayed, $30 \mathrm{mg} \mathrm{L}^{-1}$, decreased ATP levels more than $50 \%$ after $48 \mathrm{~h}$ of incubation. Copper reduced ACP activity above $145 \mathrm{mg} \mathrm{L}^{-1}$, but the two lower concentrations of 30 and $65 \mathrm{mg} \mathrm{L}^{-1}$ stimulated ACP activity.

Zinc also decreased ATP levels more than $50 \%$ after $48 \mathrm{~h}$ of incubation with the lowest concentration used $15 \mathrm{mg} \mathrm{L}^{-1}$. ACP activity was strongly reduced by $45 \mathrm{mg} \mathrm{L}^{-1}$ of zinc, but almost not affected by $15 \mathrm{mg} \mathrm{L}^{-1}$.

Triton X-100 reduced ATP levels and, less drastically, ACP activity above the lowest concentration assayed, $12.5 \mathrm{mg} \mathrm{L}^{-1}$.

Cycloheximide strongly reduced ATP levels above $0.01 \mathrm{mg} \mathrm{L}^{-1} ; 0.001 \mathrm{mg} \mathrm{L}^{-1}$ cycloheximide only slightly affected it and the lowest concentration employed, $0.0002 \mathrm{mg} \mathrm{L}^{-1}$, promoted an increase in ATP content. ACP activity was stimulated by $0.0002 \mathrm{mg} \mathrm{L}^{-1}$, but $0.001 \mathrm{mg} \mathrm{L}^{-1}$ cycloheximide did not significantly change ACP activity compared with the control conditions. Above $0.01 \mathrm{mg} \mathrm{L}^{-1}$ cycloheximide, ACP activity was reduced and with $1 \mathrm{mg} \mathrm{L}^{-1}$ completely inhibited.

Fig. 2 shows the correlation existing between the variations in ATP content and ACP activity of the cultures, after exposure to the toxic compounds. Table 2 presents the calculated values of Kendall's coefficient of 

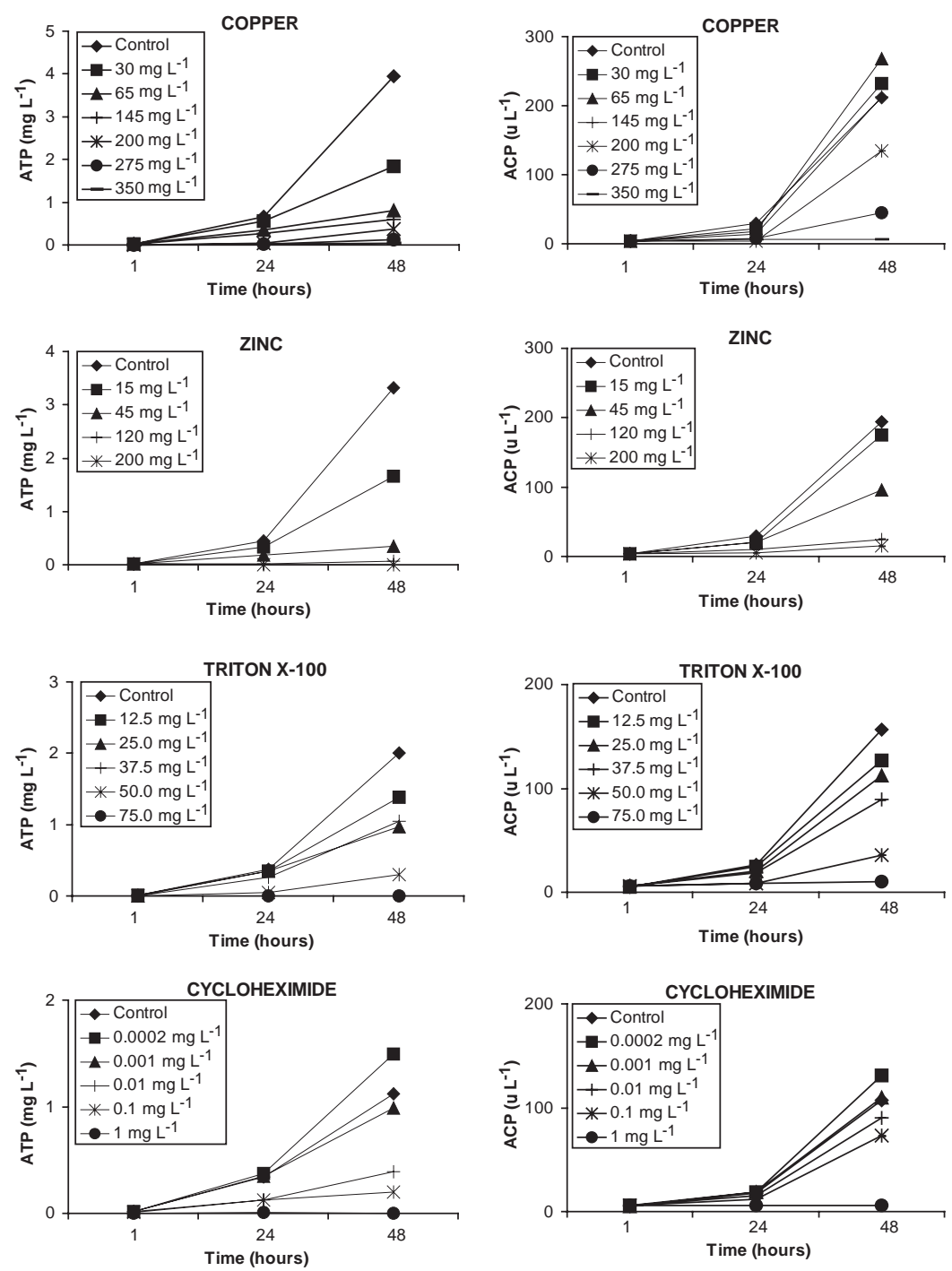

Fig. 1. Variation of ATP content and ACP activity in cultures of T. pyriformis exposed to toxicants for $48 \mathrm{~h}$, in one of the two independent assays. The results of the other assay presented the same pattern. Values are the averages of three subsamples and standard deviations are overlapped by the symbols.

rank correlation between the two variables, all of them significant at the $1 \%$ level $(P \leqslant 0.01)$.

\section{Discussion}

Zinc and Triton X-100 affected T. pyriformis negatively, causing strong decreases in ATP content and inhibition of ACP activity in the cultures at all the assayed concentrations. Other studies support these results, having observed inhibition of growth, of grazing, and of metabolic reduction of MTT in cultures of $T$. pyriformis by these toxicants, at these concentrations (Nicolau et al., 1999; Dias et al., 1999). Significant values of Kendall's coefficient of correlation between the ACP activity and the ATP content indicate that zinc and Triton X-100 affect, in the same way, these two parameters in the range of concentrations assayed.
Correlation between the two variables is almost perfect in the case of Triton X-100, all data falling near a single line. A positive correlation of these two parameters was expected: energy is needed for the phagocytosis of food molecules, for anabolism, for the synthesis of enzymes, and for the activity of these enzymes.

Copper, in contrast, decreased ATP content of the cultures at all the assayed concentrations, but the two lowest, 30 and $65 \mathrm{mg} \mathrm{L}^{-1}$, stimulated ACP activity. Nilsson (1981) mentioned the stimulation of phagocytosis on Tetrahymena by copper. These were also the concentrations that stimulated grazing activity in a previous study (Nicolau et al., 1999). This coincidence is quite interesting as ACP activity reflects the lysosomal function and, therefore, intracellular digestion in ciliated protozoa: they ingest food molecules by endocytosis from the environment in membrane-limited vacuoles (food vacuoles or phagosomes); after ingestion, these are 

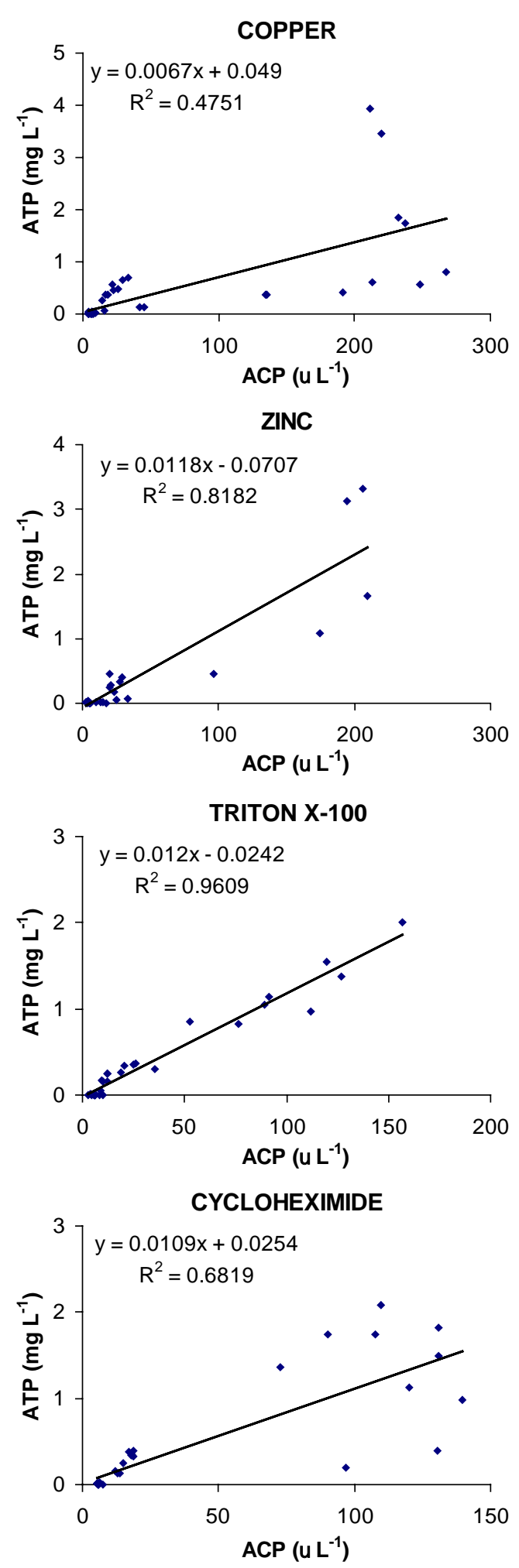

Fig. 2. Scattergram of the variations in ATP content and ACP activity in cultures of T. pyriformis exposed to toxicants for $48 \mathrm{~h}$, in one of the two independent assays. The results of the other assay presented the same pattern.

fused with one or more lysosomes and the hydrolytic enzymes of the latter then digest the content of the newly formed phagolysosome. Small-molecular-weight pro-
Table 2

Kendall's coefficient of rank correlation calculated for the variations of ATP content and ACP activity, in one of two independent assays

\begin{tabular}{ll}
\hline Toxicant & Kendall's coefficient of rank correlation \\
\hline Copper & 0.699, significant at 0.01 level, $n=43$ \\
Zinc & 0.532, significant at 0.01 level, $n=30$ \\
Triton X-100 & 0.669, significant at 0.01 level, $n=36$ \\
Cycloheximide & 0.600, significant at 0.01 level, $n=36$ \\
\hline
\end{tabular}

The results of the other assay were also correlated at 0.01 level.

ducts of digestion can then pass through the membrane of the phagolysosome and be utilized by the cell. On the other hand, one might be surprised by the decrease in ATP content of the cultures, at these two concentrations, meaning that there is a lower energy level available for increased grazing and ACP activities. A study of longer exposure times would be of great interest to find out the long-term consequences of an ATP content decrease on other cellular functions, such as grazing and ACP activity. This discrepancy is reflected in the scattergram, in which high values of ACP activity correspond to low as well as to high values of ATP content. Nevertheless it shows an unequivocal trend and a positive slope in the scattergram, and the correlation coefficient is also significant at the $1 \%$ level.

Finally, cycloheximide stimulated both ATP content and ACP activity of the cultures at $0.0002 \mathrm{mg} \mathrm{L}^{-1}$, whereas $0.001 \mathrm{mg} \mathrm{L}^{-1}$ of this compound decreased ATP content but did not significantly alter ACP activity. This might mean that the above concentration level is lying in the frontier between the two effects, functioning like a transition level between the range of low concentrations that promote stimulation and the range of high concentrations that cause inhibitory effects. The positive slope and elliptical outline of the scattergram are quite evident, and although the points of the right half of the graph are somewhat spread out, Kendall's coefficient of rank correlation is still significant at $1 \%$, revealing a clear correlation of ATP content and ACP activity.

There are several studies on the toxicity of heavy metals on T. pyriformis. Yamaguchi et al. (1973) observe complete inhibition of growth of $T$. pyriformis in axenic cultures at $1 \mathrm{mM}$ copper approximately $63.5 \mathrm{mg} \mathrm{L}^{-1}$. In contrast, Piccinni et al. (1987) observed no effect on growth following addition of copper to axenic cultures of $T$. pyriformis at a concentration of $10 \mathrm{mg} \mathrm{L}^{-1}$. Nilsson (1981) states that the addition of $100 \mathrm{mg} \mathrm{L}^{-1}$ copper (nominal concentration) to the normal $2 \%$ proteose peptone medium is tolerated by Tetrahymena and stimulates phagocytosis; cell proliferation is observed to resume after a lag period, but at a decreased rate.

In the study carried on using $T$. pyriformis under the same conditions as in the present work (Nicolau et al., 1999), copper drastically affected $T$. pyriformis above a 
concentration of $200 \mathrm{mg} \mathrm{L}^{-1}$ with 3- or 4.5-fold increases in cell numbering in $48 \mathrm{~h}$, mortality of almost $20 \%$, and significant inhibition of grazing; at a concentration of $145 \mathrm{mg} \mathrm{L}^{-1}$, there was almost no mortality, but growth and grazing were still heavily inhibited; lower concentrations inhibited growth but stimulated grazing activity and caused no mortality. Zinc caused drastic inhibition of growth and grazing and high mortality of $T$. pyriformis above $45 \mathrm{mg} \mathrm{L}^{-1}$. In the same study, Triton $\mathrm{X}-100$ disrupted all cell membranes above $50 \mathrm{mg} \mathrm{L}^{-1}$. At concentrations around $37.5 \mathrm{mg} \mathrm{L}^{-1}$, effects on individual cells varied, with growth of the more resistant cells, but long exposure times finally killed all cells. Below $25 \mathrm{mg} \mathrm{L}^{-1}$, Triton X-100 affected only growth and grazing and caused no mortality. These results were in accordance with results of other studies (Dias and Lima, 2002; Dias et al., 2003).

Studies on cycloheximide toxicity on ciliates are scarce, making comparisons difficult. Nicolau et al. (1999) and Dias et al. (1999) report only inhibitory effects in this range of concentrations, Robert and Orias (1973) state that $10.0 \mathrm{mg} \mathrm{L}^{-1}$ cycloheximide caused complete inhibition of growth and eventual lysis of $T$. pyriformis cells but do not refer to the effects of lower concentrations, and, finally, Millis and Suyama (1972) report that $1 \mathrm{mM}$ cycloheximide (which corresponds to $281.4 \mathrm{mg} \mathrm{L}^{-1}$ ) completely abolished mitochondrial ribosome synthesis, but not mitochondrial protein synthesis capacity. The fact that cycloheximide is a toxicant to eukaryotic organisms by inhibiting protein synthesis at the $80 \mathrm{~S}$ ribosome level but not $70 \mathrm{~S}$ ribosomes can complicate the interpretation of data, especially at lower concentrations, at which the toxic effects are not so drastic.

In the assays of ATP content, standard deviations above $10 \%$ appeared only in the case of $0.001 \mathrm{mg} \mathrm{L}^{-1}$ cycloheximide and this might be due to the effect of the concentration itself rather than to a methodology problem: the lower concentration of $0.0002 \mathrm{mg} \mathrm{L}^{-1}$ cycloheximide had a stimulatory effect upon this parameter and the higher concentration of $0.01 \mathrm{mg} \mathrm{L}^{-1}$ cycloheximide caused a reduction in ATP content of the cultures. In the assays of ACP activity, the standard deviations were always below $1 \%$ and in most cases below $0.1 \%$; this fact emphasizes the sensitivity of the method.

The data indicate that zinc is more toxic than copper for $T$. pyriformis, under the tested conditions, in contrast to other studies, in which copper is systematically considered more toxic than zinc to protozoa (Madoni et al., 1992, 1994, 1996), to bacterial communities of activated sludge (Cimino and Caristi, 1990), or even to algae.

Though the use of ciliates in toxicological tests is gaining the interest of an increasing number of investigators and their potential in standard bioassays has been clearly demonstrated, studies on the influence of toxicants on the energetic state of the cell by using protozoa were not found. There are several studies on hydrolase activity, namely ACP, but they aim at the knowledge of lysosomal physiology rather than to study toxic effects, and none used heavy metals, cycloheximide, or surfactant agents as toxicants (Rothstein and Blum, 1974a, b; Ricketts and Rappit, 1975; Williams and Juo, 1976). Martin and Clydes (1991), using epithelial human cells and one rat fibroblast cell line, compared some traditional methods for assessing toxicity of drugs and other chemicals to cells in culture with the ACP method. Though all methods proved to be very useful in toxicity testing, according to the authors, the ACP method gave results comparable to those of the other methods with the advantages of being faster, simpler to perform, and more accurate than other commonly used assays.

Recent work with $T$. pyriformis or other ciliates aimed, in most cases, to study the toxicological effects on mortality, growth, or phagocytosis. This work, based on two physiological responses of ciliates to toxicants, increases the current knowledge on the subject.

On the other hand, the two parameters are determined per volume unit and not per cell because the aim of this work was also to get to know if the determination of ATP content and ACP activity per volume unit of the culture of $T$. pyriformis would be sensitive enough to reflect the toxicity of several doses of copper, zinc, Triton X-100, and cycloheximide. No need to determine the cell density has the advantage of eliminating a step that is always time consuming and expertise exigent. This will not prevent the need for determining the ATP content and the ACP activity per cell before the eventual implementation of such a test to evaluate toxicity. The two assays were easy to carry out and did not demand particular skills or expertise and they seem to be promising alternatives to other conventional toxicological tests using suspension cultures.

\section{Acknowledgments}

Ana Nicolau was supported by Grant BPD/11594/02 from PRAXIS XXI.

\section{References}

Akers, K.S., Sinks, G.D., Schultz, T.W., 1999. Structure-toxicity relationships for selected halogenated aliphatic chemicals. Environ. Toxicol. Pharmacol. 7, 33-39.

Cimino, G., Caristi, C., 1990. Acute toxicity of heavy metals to aerobic digestion of waste cheese whey. Biol. Wastes 33, 201-210.

Dias, N., Lima, N., 2002. A comparative study using a fluorescence based and a direct-count assay to determine cytotoxicity in Tetrahymena pyriformis. Res. Microbiol. 153, 313-322. 
Dias, N., Nicolau, A., Lima, N., Carvalho, G.S., Mota, M., 1998. The use of immunocytochemical methods for toxicity assessment in Tetrahymena pyriformis. Cuad. Invest. Biol. (Bilbao) 20, 75-78.

Dias, N., Nicolau, A., Carvalho, G.S., Mota, M., Lima, N., 1999. Miniaturization and application of the MTT assay to evaluate metabolic activity of protozoa in the presence of toxicants. J. Basic Microbiol. 39, 103-108.

Dias, N., Mortara, A., Lima, N., 2003. Morphological and physiological changes in Tetrahymena pyriformis for the in vitro cytotoxicity assessment of Triton X-100. Toxicol. In vitro 17, 357-366.

Dowhanick, T.M., Sobczak, J., 1994. ATP bioluminescent procedure for viability testing of potential beer spoilage microorganisms. J. Am. Soc. Brew. Chem. 52, 19-23.

Gamborg, G., Hansen, E.H., 1994. Flow-injection bioluminescent determination of ATP on the use of the luciferin-luciferase system. Anal. Chim. Acta 285, 321-328.

Gikas, P., Livingston, A.G., 1993. Use of ATP to characterize biomass viability in suspended and immobilized bioreactors. Biotechnol. Bioeng. 42, 1337-1351.

Madoni, P., Esteban, G., Gorbi, G., 1992. Acute toxicity of cadmium, copper, mercury and zinc to ciliates from activated sludge plants. Bull. Environ. Contam. Toxicol. 49, 900-905.

Madoni, P., Davoli, D., Gorbi, G., 1994. Acute toxicity of lead, chromium and other heavy metals to ciliates from activated sludge plants. Bull. Environ. Contam. Toxicol. 53, 420-425.

Madoni, P., Davoli, D., Gorbi, G., Vescovi, L., 1996. Toxic effects of heavy metals on the activated sludge protozoan community. Water Res. 30, 135-141.

Martin, A., Clydes, M., 1991. Acid phosphatase endpoint for in vitro toxicity tests. In vitro Cell. Dev. Biol. 27A, 183-184.

Meyer, R.B., Boyd, C.R., Rein, D.C., Keller, S.J., 1971. Effects of ethidium bromide on growth and morphology of Tetrahymena pyriformis. Exp. Cell Res. 70, 233-237.

Millis, A.J.T., Suyama, Y., 1972. Effects of chloramphenicol and cycloheximide on the biosynthesis of mitochondrial ribosomes in Tetrahymena. J. Biol. Chem. 247, 4063-4073.

Nicolau, A., Mota, M., Lima, N., 1999. Physiological responses of Tetrahymena pyriformis to copper, zinc, cycloheximide and Triton X-100. FEMS Microbiol. Ecol. 30, 209-216.

Nicolau, A., Dias, N., Mota, M., Lima, N., 2001. Trends on the use of protozoa in the assessment of wastewater treatment. Res. Microbiol. 152, 621-630.
Nilsson, J.R., 1981. Effects of copper on phagocytosis in Tetrahymena. Protoplasma 109, 359-370.

Nilsson, J.R., 1989. Tetrahymena in cytotoxicology: with special reference to effects of heavy metals and selected drugs. Eur. J. Protistol. 25, 2-25.

Noever, D.A., Matsos, H.C., Cronise, R.J., Looger, L.L., Relwani, R.A., 1994. Computerized in vitro test for chemical toxicity on Tetrahymena swimming patterns. Chemosphere 29, 1373-1384.

Piccinni, E., Irato, P., Coppelloti, O., Guidolin, L., 1987. Biochemical and ultrastructural data on Tetrahymena pyriformis treated with copper and cadmium. J. Cell Sci. 88, 283-293.

Ricketts, T.R., Rappit, A.F., 1975. The adaptative acid phosphatase of Tetrahymena pyriformis GL-9. Effect of endocytosis of various nutrients. Protoplasma 85, 119-125.

Robert, C.T., Orias, E., 1973. A cycloheximide mutant of Tetrahymena pyriformis. Exp. Cell Res. 81, 312-316.

Rothstein, R.L., Blum, J.J., 1974a. Lysosomal physiology in Tetrahymena. II. Effect of culture age and temperature on the extracellular release of 3 acid hydrolases. J. Protozool. 21, 163-168.

Rothstein, R.L., Blum, J.J., 1974b. Lysosomal physiology in Tetrahymena. III. Pharmacological studies on acid hydrolase. J. Cell Biol. 62, 844-859.

Sauvant, M.P., Pepin, D., Bohatier, J., Groliere, C.A., 1994. Comparative study of two in vitro models (L-929 fibroblasts and Tetrahymena pyriformis GL) for the cytotoxicological evaluation of package water. Sci. Total Environ. 156, 159-167.

Twagilimana, L., Bohatier, J., Groliere, C.A., Bonnemoy, F., Sargos, D., 1998. A new low-cost microbiotest with the protozoan Spirostomum teres: culture conditions and assessment of sensitivity of the ciliate to 14 pure chemicals. Ecotoxicol. Environ. Saf. 41, 231-244.

Ward, C.J., Codd, G.A., 1999. Comparative toxicity of four microcystins of different hydrophobicities to the protozoan Tetrahymena pyriformis. J. Appl. Microbiol. 86, 874-882.

Williams, J.T., Juo, P.S., 1976. Release and activation of a particulate bound acid phosphatase from Tetrahymena pyriformis. Biochim. Biophys. Acta 422, 120-126.

Yamaguchi, N., Wada, O., Ono, T., Takasi, K., Toyokawa, K., 1973. Detection of heavy metal toxicity by Tetrahymena pyriformis culture method. Ind. Health 11, 27-31.

Yoshioka, Y., Ose, Y., Sato, T., 1985. Testing for the toxicity of chemicals with Tetrahymena pyriformis. Sci. Total. Environ. 43, $149-157$. 